\title{
Vivências de idosos alcoolistas: contribuições para a enfermagem gerontológica
}

\author{
Lives of alcoholic older adults: contributions to gerontological nursing \\ Vivencia de ancianos alcohólicos: contribuciones a la enfermera gerontológica
}

\author{
Rejane de Fátima Parada Viegas'; Janaina Moreno de Siqueira"; Marilurde Donato"I'; \\ Maria Yvone Chaves Mauro ${ }^{\prime v}$; Sheila Nascimento Pereira de Farias ${ }^{v}$; Beatriz Gomes da Silva ${ }^{\text {vl }}$
}

\begin{abstract}
RESUMO:
Objetivo: identificar as causas do uso abusivo de bebidas alcoólicas por idosos. Método: pesquisa qualitativa, utilizou-se o método história de vida para relatar vivência de idosos alcoolista. A amostra foi composta de 22 idosos, a coleta de dados foi realizada em 2007 e 2008 , em um albergue público de Campo Grande e três grupos de AA de Jacarepaguá, todos em bairros do Rio de Janeiro. O projeto foi aprovado por Comitê de Ética em Pesquisa. As entrevistas foram gravadas em mp3 e na análise de dados, procedeu-se a transcrição das gravações dos depoimentos. Resultados: dos depoimentos emergiram a categoria - os bastidores do alcoolismo na velhice e as subcategorias - a família como causa primária e a influência social. Conclusão: é necessário implementar política de educação e de saúde para prevenção, tratamento e reabilitação do alcoolismo, também entre idosos, visando à redução de danos e melhor qualidade de vida.

Descritores: Idoso; alcoolista; vivência; enfermagem.
\end{abstract}

\section{ABSTRACT:}

Objective: to identify the causes of abusive use of alcoholic beverages by the elderly. Method: in this qualitative study, the life history method was used to report the experiences of a sample of 22 older adults. Data were collected in 2007 and 2008 at a public hostel in Campo Grande and from three AA groups in Jacarepaguá, all neighborhoods of Rio de Janeiro City. The project was approved by the research ethics committee. The interviews were recorded in $\mathrm{mp} 3$ and, for data analysis purposes, the recordings of the testimonies were transcribed. Results: the category that emerged from the testimonies was: behind the scenes of alcoholism in old age; and the subcategories: the family as primary cause and social influence. Conclusion: there is a need to implement education and health policy providing for the prevention, treatment and rehabilitation of alcoholism, also among older adults, with a view to harm reduction and better quality of life.

Descriptors: Elderly; alcoholic; living experience; nursing.

\section{RESUMEN:}

Objetivo: identificar las causas del uso abusivo de bebidas alcohólicas por los ancianos. Método: investigación cualitativa en la que se utilizó el método de la historia de vida para relatar la experiencia de ancianos alcohólicos. La muestra se compuso de 22 ancianos, la recolección de datos tuvo lugar en 2007 y 2008, en un albergue público de Campo Grande y tres grupos de AA de Jacarepaguá, todos ubicados en barrios de Río de Janeiro. El proyecto fue aprobado por el Comité de ética de la investigación. Las entrevistas se registraron en MP3 y, en el análisis de datos, se realizó la transcripción de las grabaciones de las declaraciones. Resultados: de las declaraciones surgieron la categoría - las trastiendas del alcoholismo en la vejez - y las subcategorías - la familia como causa primaria y la influencia social. Conclusión: es necesario implementar políticas de educación y salud para la prevención, el tratamiento y la rehabilitación del alcohólico, también entre los ancianos, con el objetivo de reducir los daños y mejorar la calidad de vida.

Descriptores: Anciano; alcohólico; vivencia; enfermería.

\section{INTRODUÇÃO}

O relatório mundial sobre envelhecimento e saúde divulgado pela Organização Mundial de Saúde (OMS), em 2015, realça que o envelhecimento saudável é mais que apenas a ausência de doença. Para a maioria dos adultos maiores, a manutenção da habilidade funcional é o mais importante.

Em decorrência, os maiores custos à sociedade não são os gastos realizados para promover esta habilidade funcional, mas sim os benefícios que poderiam ser perdi- dos se não fossem realizadas adaptações e investimentos necessários. O enfoque social recomendado para abordar o envelhecimento da população, que inclui a meta de construir um mundo favorável aos adultos maiores, requer uma transformação dos sistemas de saúde que substitua os modelos curativos baseados na doença pela prestação de atenção integrada e centrada nas necessidades ${ }^{1}$.

Assim, com o crescimento da população idosa², mudanças drásticas precisam acontecer nos sistemas

'Mestre. Universidade Federal do Rio de Janeiro. Escola de Enfermagem Anna Nery. Rio de Janeiro (RJ), Brasil. E-mail: rejaneparada@gmail.com "Pós-Graduada. Universidade Federal do Rio de Janeiro, Escola de Enfermagem Anna Nery. Rio de Janeiro (RJ), Brasil. E-mail: janaina.moreno@ymail.com '"Doutora. Universidade Federal do Rio de Janeiro. Escola de Enfermagem Anna Nery. Rio de Janeiro (RJ), Brasil. E-mail: marilurdedonato@terra.com.br IVDoutora. Universidade do Estado do Rio de Janeiro. Faculdade de Enfermagem UERJ. Rio de Janeiro (RJ), Brasil. E-mail: mycmauro@gmail.com vDoutora. Universidade Federal do Rio de Janeiro. Escola de Enfermagem Anna Nery. Rio de Janeiro (RJ), Brasil. E-mail: sheilaguadagnini@gmail.com.br VIEspecialista. Universidade Federal do Rio de Janeiro, Escola de Enfermagem Anna Nery. Rio de Janeiro (RJ), Brasil. E-mail: beatrizg.ufrj@gmail.com 
de saúde, para que se possam implantar medidas que garantam, apoiem e incentivem uma longevidade saudável ${ }^{3}$. Por outro lado, concomitantemente com o aumento da população idosa, temos uma cultura de ingestão de bebidas alcoólicas bastante difundidas.

Nesse sentido, o Brasil encontra-se na $49^{a}$ posição entre os 193 países avaliados pelo Centro de Informações sobre Saúde e Álcool (CISA), revelando um aumento de ingestão do álcool da ordem de 43,5 \% em 10 anos, superando a média. Assim, elevaram-se as desordens relacionadas ao uso do álcool no país, representando $63 \%$ dos transtornos emergentes, $60 \%$ dos índices de cirrose hepática e a 18,5\% dos acidentes de trânsito ${ }^{4}$.

A Organização Mundial de Saúde (OMS), em Assembleia Mundial da Saúde realizada em 2010, chegou ao consenso sobre a necessidade de se criar uma estratégia global para enfrentar os problemas gerados pelo álcool ${ }^{5}$. O uso abusivo dessa substância está relacionado ao óbito de $5,9 \%$ de pessoas no mundo ${ }^{6}$.

Com base nessa realidade, o estudo teve como objeto o uso abusivo de bebida alcoólica por pessoas idosas e como principal questão norteadora: o que leva o idoso a se tornar alcoolista?

Assim, foi estabelecido como objetivo: identificar as causas do uso abusivo de bebidas alcoólicas por idosos.

\section{REVISÃo DE LITERATURA}

Um estudo relevante apontado pelo Instituto Brasileiro de Geografia e Estatística (IBGE) mostrou que o número de idosos cresce e ultrapassa a marca de 30 milhões. Esses dados epidemiológicos foram registrados no período de 2012 a 2017 e mostram que no Brasil, houve um aumento da população idosa em maior proporção nos estados do Rio de Janeiro e em Porto Alegre, ambos com $18,6 \%$ de pessoas na faixa etária de 60 anos ou mais ${ }^{7}$.

Além disso, um estudo realizado pelo CISA em 2018 evidenciou que $27 \%$ dos idosos entrevistados consumiam cerveja, $21 \%$ bebidas destiladas e $35 \%$ bebiam com frequência, sendo o consumo mais prevalente em homens do que em mulheres ${ }^{8}$. Esta estatística de aumento da ingestão de álcool por idosos corrobora outro estudo realizado para avaliar a associação entre o consumo de álcool e a qualidade de vida, onde a prevalência de consumo de álcool foi de $8,9 \%$ na faixa etária de 70 a 79 anos, com presença de depressão ${ }^{9}$.

Outro documento de relevo, é uma matéria publicada em 2017 pelo hospital sírio-libanês sobre o consumo de álcool em idosos. Afirma que o alcoolismo nesse grupo está associado à aposentadoria, solidão, viuvez, isolamento social e a algumas doenças crônicas ${ }^{10}$, o que pode ser corroborado por um estudo realizado na Paraíba, que afirma que o uso excessivo do álcool em idosos é consequência das transformações vivenciadas principalmente relacionadas aos aspectos sociais ${ }^{11}$.
Por fim, existe maior risco de vulnerabilidade para problemas físicos, psicológicos e sociais devido ao abuso de álcool pelos idosos que nem sempre são percebidos pelos profissionais de saúde e, desta forma, as doenças são agravadas pelo alcoolismo acarretando grande desafio para os serviços de saúde ${ }^{12}$.

\section{Metodologia}

Trata-se de um estudo de natureza qualitativa, realizado entre o segundo semestre de 2007 e o primeiro semestre de 2008. O método utilizado foi o de história de vida, no qual é considerado o relato da pessoa que vivenciou a experiência como fonte dos dados, não cabendo ao pesquisador interferir, induzir ou questionar a história contada ${ }^{13}$.

Para que fosse possível compreender o fenômeno da experiência vivenciada por cada idoso e levando em consideração as diferenças no contexto social de cada um, foram escolhidos dois cenários de forma a evidenciar diversidade de vivências. São eles: uma Instituição Pública que abriga população de rua no bairro de Campo Grande e três grupos de Alcoólicos Anônimos (AA) no bairro de Jacarepaguá, no Município do Rio de Janeiro.

Foram participantes da pesquisa idosos alcoolistas de ambos os sexos, sendo 10 moradores do albergue público e 12 frequentadores das reuniões dos Alcoólicos Anônimos (AA). Os critérios de inclusão foram: ter mais de sessenta anos, ser alcoolista e ter capacidade cognitiva para responder a pergunta de pesquisa.

Para a presente pesquisa foram utilizados dois formulários, um para a caracterização dos depoentes, contendo variáveis sociodemográficas (cor, idade, quantidade de filhos, estado civil, profissão, escolaridade, situação de trabalho, instituição de origem, idade em que começou a beber, tempo de consumo, status atual da utilização de bebida alcoólica) e de saúde (portador de hipertensão ou diabetes, e se sofreu algum acidente depois de ter ingerido álcool); e outro com o seguinte comando para extração dos dados relatados pelos participantes: fale o que o senhor considera importante a respeito da sua vida e que tenha relação com o uso abusivo de bebida alcoólica.

Para verificar a adequação do comando foram realizadas duas entrevistas-piloto, sendo a primeira com um residente do Albergue e a outra com participantes do AA. Previamente às entrevistas, foi apresentado a cada idoso o Termo de Consentimento Livre e Esclarecido (TCLE) previsto na Resolução no 196/96 do Conselho Nacional de Ética em Pesquisa - CONEP vigente à época da coleta de dados, documento que visava assegurar o sigilo e o anonimato. Este documento foi assinado por todos os que decidiram participar do estudo e que, doravante, passaram a ser considerados depoentes. Foi utilizada a letra $E$ (entrevistado) seguida do número de ordem da entrevista de modo a preservar o anonimato dos depoentes. 
As entrevistas foram gravadas em MP3 sob a justificativa de garantir a fidedignidade do que fosse dito e no momento da análise dos dados procedeu-se à transcrição das gravações. Cada entrevista durou, em média, noventa minutos, pela dificuldade dos idosos em se expressarem. É válido ressaltar que antes de iniciá-las, reiteravam-se a cada depoente os esclarecimentos sobre o assunto investigado, bem como sobre o conteúdo do TCLE.

Foram registradas em diário de campo todas as expressões corporais dos participantes, durante a entrevista, assegurando também o registro daquilo que não foi dito a fim de não se perder nenhum detalhe. A partir dos depoimentos registrados, realizou-se a análise de conteúdo emergindo categorias e subcategorias que permitiram a interpretação e compreensão do tema de pesquisa.

\section{RESUlTADOS E Discussão}

Como primeira constatação, foi observado um baixo nível de escolaridade entre os participantes, onde apenas um concluiu nível superior, quatro o ensino médio e os demais tinham escolaridade inferior as citadas, sendo cinco analfabetos.

Ademais, na amostra, dos dez que estavam aposentados, oito ainda trabalhavam para complementar a renda, sendo um pensionista.

Em relação ao tempo aproximado de consumo alcoólico, nove começaram a beber na infância, seis na adolescência, cinco na fase adulta e somente dois não souberam informar.

Quanto às doenças crônicas, sete disseram ser hipertensos e três diabéticos, sendo o uso excessivo de álcool um possível agravante da situação de saúde desses indivíduos culminando no aparecimento dessas morbidades.

A faixa etária do grupo pesquisado variou entre 60 e 78 anos, no qual treze tinham de 60 a 70 anos e nove 71 anos ou mais.

Dos vinte e dois depoentes, quinze eram brancos, quatro pardos e dois negros.

No tocante ao número de filhos, dezesseis idosos disseram ter de um a cinco filhos, cinco informaram que não tiveram filhos e apenas um relatou ter seis filhos.

Dentre os depoentes, sete relataram ser casados, sete separados, quatro solteiros, dois divorciados e dois viúvos. Os que relataram nunca terem casado, eram moradores do albergue público, originários de famílias desestruturadas, marcadas pela violência, miséria, fome, maus tratos e, conforme seus relatos residiam em outros Estados, mas evadiram-se de suas casas ainda na adolescência com o objetivo de ganhar o próprio sustento no município do Rio de Janeiro, sem obterem sucesso.

Em última instância, tanto as dificuldades vividas ao longo da vida, como as relações discriminatórias foram autorreferidas pelos idosos como desencadeadoras do uso excessivo de álcool.

\section{Categoria e subcategorias do estudo}

Da categoria: Os bastidores do alcoolismo na velhice surgiram as subcategorias analisadas a seguir.

\section{Subcategoria 1: a família como causa primária}

Constata-se que dos vinte dois depoentes, doze eram membros do AA e os outros dez, moradores de um albergue público. No primeiro caso, todos tinham família que os apoiavam; no segundo, eram indigentes, sendo que alguns até frequentavam as reuniões do AA.

Dentre os fatores de consumo excessivo de álcool tem-se a desestruturação familiar, como pais separados, conflitos no lar, muita permissividade, maus tratos, ausência de religião e convívio com parentes que utilizam drogas (adictos) ou que consomem álcool em excesso ${ }^{14}$.

O uso abusivo do álcool oportuniza a fragmentação da família, tendo em vista que, por vezes, está associado à violência e maus tratos, o que pode progredir para a separação de uma família ${ }^{15}$.

Meu pai, como eu falei no início, era separado, meu pai sumia, não tinha responsabilidade [...], hoje eu sei que era portador do alcoolismo, bebia muito [...], esquece de trabalhar também [...] tenho um filho mais velho e um de vinte anos que também são portadores da doença [...]. (E1)

[...] sempre ia buscar uma cachacinha lá para minha mãe, que gostava de beber escondido do meu pai [...]. (E16)

[...] foi em 78, eu estava ajudando vizinhos [...] quando vieram me avisar: Oh! Corre que teu pai matou tua mãe! [...] quando cheguei lá, o chão todo ensanguentado [...] aí fomos no barzinho, ele todo ensanguentado, tomando uma cachaça[...] de lá para cá eu engrenei nela direto [...]. (E18)

No relato de E18, observa-se como causa de uma exposição crônica à bebida, uma situação aguda estressante que o levou para o alcoolismo. Dentre as finalidades para utilização da bebida alcóolica, por vezes os indivíduos a ingerem para fugir da realidade de suas vidas, tentando mascarar com a bebida períodos de sofrimento intenso, tendo em vista as propriedades excitatórias do álcool que fornece, inicialmente, sensação de prazer e euforia ${ }^{16}$.

Comecei a beber com dez anos de idade. Minha mãe lavava roupa para fora aí pedia para mim comprar bebida [...] botava a bebida embaixo do tanque[...] quando ia para a cozinha eu bebia[...]. (E18).

Eu me tornei alcoólica porque meu pai era um ditador, eu tinha bronca dele[...] nunca vi ele brigando com minha mãe, também não podia, porque minha mãe era assim mais submissa, de dar nojo sabe [...] eu tinha raiva porque dominava todos os meus irmãos, [...] queria ser a salvadora (E9).

Analisando esta fala, é possível observar como a depoente em questão demonstrava a sua reprovação por ver seu referencial de mulher fracassado, dominado, 
mal sucedido, destacando ainda que quando fazia algo errado, seu pai ordenava, como castigo, que sua mãe a surrasse nas pernas com o fio do ferro elétrico. Seus irmãos, embora bem mais velhos, eram dominados por seu pai e ninguém, além dela, era capaz de contrariá-lo.

A família é o nosso principal ponto socializador, é através dela que temos o primeiro contato com as pessoas e ela pode influenciar positiva ou negativamente seus membros. Nos relatos acima, podemos perceber como o comportamento dos pais refletiu na saúde mental, física e financeira do lar, contribuindo para que os filhos se tonassem alcoolistas no futuro ${ }^{17}$.

Dentre os motivos que levam o indivíduo a beber, o ambiente familiar é um dos fatores que favorecem o consumo, juntamente com os benefícios que o álcool trás em relação à socialização e à interação no local de trabalho ${ }^{18}$.

\section{Subcategoria 2: A influência da sociedade}

A necessidade de socialização aumenta a prevalência de ingestão de álcool, sendo importante levar em conta os fatores sociodemográficos para que políticas e programas de prevenção possam ser implementados ${ }^{9}$.

Aqui foi levado em consideração o convívio dos idosos com pessoas que bebiam em quaisquer ambientes e os fatores que influenciavam o consumo de bebidas.

[...] fui trabalhar em um ambiente onde todas as pessoas bebiam [...] eles tinham o hábito de beber na hora do almoço[...] e eu, para me integrar, [...] para fazer parte daquele meio [...] depois passamos a beber fora do horário do expediente [...] para fazer o balanço do dia. (E14)

O depoimento mostra que o indivíduo constrói valores baseando-se naquilo que seu grupo representa; assim, sua identidade é projetada em práticas culturais que o levam a adquirir uma determinada imagem na sociedade. Muitos indivíduos passam a reproduzir os hábitos de um grupo para preservarem sua participação no convívio social, de forma a não se sentirem sós. Outros, para evitar ser motivos de piadas do grupo por não reproduzir algo que é aceito, incentivado e praticado pelas pessoas do convívio, sendo quase uma condição para aceitação do indivíduo na sociedade.

O comportamento individual pode influenciar seus pares de forma complexa, tendendo a perpetuar o hábito de beber por todo um grupo, sendo mais difícil resistir quando um deles tentar modificar seu padrão de consumo e hábito ${ }^{19}$.

Um estudo realizado na cidade do Rio de Janeiro sobre motivação para beber, aponta que de 21 entrevistados, oito sofrem influência dos amigos para consumir álcool, em relação à finalidade, cinco disseram que bebem para acompanhar amigo, sendo a bebida alcoólica um facilitador de integração social ${ }^{20}$.

Neste estudo, muitos depoentes relataram evitar ir à festas ou locais que servissem bebidas alcoólicas, e até mesmo a companhia de pessoas que tivessem o hábito de beber, para fugir da influência que o ambiente e pessoas tem no consumo do álcool conforme relato abaixo:

Lembro que comecei a trabalhar, e conviver com pessoas na fábrica, que também tinham problemas de alcoolismo. Eram farras homéricas[...] eu evitava até ir a algumas festas, ir em reuniões de algumas famílias, os meus amigos namorados das minhas irmãs, também tinham esse hábito de gostar de beber, então eu estava no meio [...]. (E14)

No depoimento a seguir, observa-se que a cultura social também influencia o hábito de beber, pois o depoente desenvolveu o hábito de ingerir bebida alcóolica por questões familiares e culturais.

[...] fui nascido e criado num lugar onde a bebida, o vinho principalmente, e a cerveja faziam parte da alimentação[...] fui criado com isso [...]. (E4)

As experiências individuais por vezes são fruto de práticas desenvolvidas por seus grupos. Os costumes e hábitos histórico-culturais de determinados grupos e/ ou regiões asseguram, de certa forma, a propagação da memória de suas práticas, o que leva à replicação por parte das pessoas inseridas nesse convívio. Assim, o ato de ingerir o álcool tem forte relação com as crenças de grupos de convívio, em especial da família. Em algumas culturas, o álcool é visto como um remédio capaz de abrir o apetite, portanto, desde a infância o indivíduo aprende com a mãe e familiares a respeito da prática desse ato antes de se alimentar. Assim, com o passar dos anos, isso se torna um hábito como algo quase intrínseco da rotina do indivíduo que propaga isso às gerações futuras. Outros povos utilizam o álcool em cerimônias, como no nascimento, casamento e morte, chegando por vezes ao estado de embriaguez.

A educação em saúde para determinados grupos pode ser um desafio, entretanto, trabalhar com a redução de danos, levando em consideração o modo de vida do indivíduo e seus saberes e práticas, pode ser o caminho para construir padrões de consumo com menos agravos à saúde ${ }^{21}$.

\section{CONCLUSÃo}

À guisa de conclusão, pode-se identificar alguns pontos bastante relevantes do estudo.

A primeira ideia é que a maioria dos depoentes começou a beber na infância ou adolescência, desta forma percebe-se uma necessidade de qualificar os profissionais nas escolas para a detecção do uso nocivo de álcool por crianças e posterior encaminhamento para os órgãos de competência. Além disso, torna-se também necessário, capacitar os profissionais de saúde a identificarem, no momento da consulta, o alcoolismo como pano de fundo do agravamento de doenças crônicas, já que os idosos não procuram os serviços de saúde para tratar o alcoolismo.

Outro ponto importante, é a implementação de políticas para realização de um acolhimento adequado aos idosos alcoolistas nos centros de convivência e nas 
instituições públicas, encaminhando-os para atendimento em centros de tratamento e reabilitação sempre que for necessário possibilitando-lhes, assim, vivenciar a velhice em condições de bem-estar.

Outrossim, instituir políticas de educação e de saúde para prevenção, tratamento e reabilitação do alcoolismo, visando à redução de danos e melhoria da qualidade de vida aos que abusam do álcool e carregam significado cultural e familiar na ingestão de bebida alcóolica, pode favorecer a aceitação das mudanças de hábitos por parte desse grupo, onde a equipe sempre deve utilizar-se da escuta ativa e da construção da educação em saúde a partir da realidade do grupo.

Sem embargo, como limitação de estudo foi realizada a pesquisa em três alcoólicos anônimos e um albergue público, podendo ser expandido para outras instituições e/ou alcoólicos anônimos para se obter informações mais diversificadas de modo a compreender melhor a reação alcoolismo/idoso de maneira mais abrangente.

Por fim, tendo em vista tudo o que foi abordado no presente artigo, recomenda-se a produção de futuros estudos que busquem compreender melhor a vivência dos alcoolistas idosos para tratamento mais efetivo.

\section{REFERÊNCIAS}

1. Organização Mundial de Saúde. Relatório de envelhecimento e saúde. Genebra: OMS; 2015.

2. Silva DM, Vilela ABA, Oliveira DC, Alves MR. A estrutura da representação social de família para idosos residentes em lares intergeracionais. Rev. enferm. UERJ. 2015; 23(1):21-6.

3. Borges CL, Silva MJ, Clares JWB, Nogueira JM, Freitas MC. Características sociodemográficas e clínicas de idosos institucionalizados: contribuiç̧̃̃es para o cuidado de enfermagem. Rev. enferm. UERJ. 2015; 23(3):381-7.

4. Centro de Informações Sobre Saúde e Álcool. Dados epidemiológicos sobre o uso de álcool no Brasil. São Paulo: CISA; 2014.

5. Organização Mundial de Saúde. Manual de Intervenções para transtornos mentais, neurológicos e por uso de álcool e outras drogas na rede de atenção básica à saúde. Genebra: WHO; 2010. 6. World Health Organization. Global strategy to reduce harmful use of alcohol. Genebra: WHO; 2012.

7. Ministério do Planejamento, Orçamento e Gestão (Br), Instituto Brasileiro de Geografia e Estatística. Agência IBGE. Notícias. Número de idosos cresce 18\% em 5 anos e ultrapassa 30 milhões em 2017. Rio de Janeiro: IBGE; 2018.

8. Centro de Informações Sobre Saúde e Álcool. Perfil do consumo de álcool por idosos brasileiros e fatores. São Paulo: CISA; c2018.

9. Soares SM, Lima EDRP, Naegle MA, Silva PAB, Santos JFG, Silva LB. Consumo de álcool e qualidade de vida em idosos na saúde da família. R. enferm. Cent.-Oeste Min. 2016;6(3): 2362-76.

10. Hanna C. Sua saúde. Consumo de álcool entre idosos aumenta. São Paulo: Hospital Sírio-Libanês; 2017.

11. Costa IP, Oliveira FKS, Pimenta CJL, Almeida MR, Moraes JCO, Costa SP. Aspectos Relacionados ao abuso e dependência de álcool por idosos. Rev. enferm. UFPE on line. 2017;11(6):2323-8. 12. Kano MY, Santos MA, Pillon SC. Uso do álcool em idosos: validação transcultural do Michigan Alcoholism Screening Test Geriatric Version (MAST-G). Esc. Enferm. USP. 2014;48(4):648-55. 13. Bertaux D. Narrativas de vida: a pesquisa e seus métodos. São Paulo: Paulus; 2010.

14. Matos AM, Carvalho RC, Costa MCO, Gomes KEPS, Santos LM. Consumo frequente de bebidas alcoólicas por adolescentes escolares: estudo de fatores associados. Rev. bras. epidemiol. 2010;13(2): 302-13.

15. Teixeira EP, Hoepers NJ, Correa SM, Dagostin VS, Soratto MT. O enfrentamento da família diante do alcoolismo. Revista saúde. com. 2015;11(3):213-26.

16. Martins KD. A dependência do álcool na dialética do envelhecimento. Rev. Cocar. 2014; 8(16):137-49.

17. Centro de Informações Sobre Saúde e Álcool. Problemas sociais decorrentes do uso do álcool. São Paulo: CISA; 2014.

18. Buss PM. Promoção da saúde e qualidade de vida. Ciênc. saúde coletiva (Online). 2000;5(1):163-77.

19. Centro de Informações Sobre Saúde e Álcool. A influência dos amigos no consumo de álcool. São Paulo: CISA; 2014.

20. Neves KC, Teixeira MLO, Ferreira MA. Fatores e motivação para o consumo de bebidas alcoólicas na adolescência. Esc. Anna Nery Rev. Enferm. 2015;19(2):286-91.

21. Bertoni LM. Alcoolismo e meio rural. Rev. GeoNordeste. 2017; 28(1):98-113. 